\title{
An example of an amenable action from geometry
}

\author{
R. J. SPATZIER \\ Department of Mathematics, State University of New York at Stony Brook, \\ Stony Brook, NY 11794, USA
}

(Received 24 November 1985 and revised 1 May 1986)

\begin{abstract}
Let $M$ be a compact manifold of not necessarily constant negative curvature. We observe that $\pi_{1}(M)$ acts amenably on the sphere at infinity of the universal cover of $M$ with respect to a natural measure class. We also note that this action is of type $\mathrm{III}_{1}$.
\end{abstract}

\section{Introduction}

In this work we describe a geometric version of the following basic example of an amenable action [ $\mathbf{Z 3}$, example 5.4]:

Let $\Gamma$ be a lattice in a connected real semisimple Lie group $G$ of the non-compact type. Let $P$ be a minimal parabolic subgroup of $G$. Then $\Gamma$ acts amenably on $G / P$ with respect to Haar measure.

If $G$ has real rank 1 then $G / P$ can be identified with the sphere at infinity [E], [BGS] of the globally symmetric space $G / K$, where $K$ is a maximal compact subgroup of $G$. Then the action of $\Gamma$ on $G / P$ becomes the natural extension of the $\Gamma$-action on $G / K$ to the sphere at infinity.

More generally, let $M$ be a compact Riemannian manifold of negative sectional curvature $K$. Set $\Gamma=\pi_{1}(M)$. Then $\Gamma$ acts on the sphere at infinity $S$ of the universal cover $\tilde{M}$ of $M$. We describe a natural measure class on $S$ (recall that in general $S$ is only a topological manifold and does not have a canonical Lebesgue measure class): if $p \in \tilde{M}$, define a map $\phi_{p}$ from the unit sphere $S_{p}$ in $T_{p} \tilde{M}$ to $S$ by $\phi_{p}(v)=\gamma_{v}(\infty)$ where $\gamma_{v}$ is the unique geodesic with $\dot{\gamma}_{v}(0)=v$. Then $\phi_{p}: S_{p} \rightarrow S$ is a homeomorphism [BGS, 3.2]. Moreover, if $q \in \tilde{M}$ then $\phi_{q}^{-1} \circ \phi_{p}: S_{p} \rightarrow S_{q}$ is absolutely continuous. Indeed if $v \in S_{p}$ and $w \in S_{1}$ then $\phi_{p}(v)=\phi_{p}(w)$ if and only if $v$ and $w$ lie on the same leaf of the weak stable foliation $W^{w s}$ of the geodesic flow. Since $S_{p}$ and $S_{q}$ are nowhere tangent to $W^{\text {ws }}$ the claim follows from the absolute continuity of $W^{\text {ws }}$ $[A, \S 5]$. Hence the image of Lebesgue measure on $S_{p}$ under $\phi_{p}$ defines a measure class on $S$ independent of $p \in M$. We denote it by $[\lambda]$.

We refer to $[\mathbf{M}]$ for the notions of amenability and type.

Proposition 1. The action of $\Gamma$ on $S$ is ergodic, amenable and of type $\mathrm{III}_{1}$ with respect to $[\lambda]$.

The third claim generalizes a result of D. Sullivan and R. J. Zimmer [Su, theorem 6], [Z3, example 5.14]. 
We reduce proposition 1 to a theorem of Bowen utilizing a certain duality between the $\Gamma$-action on $S$ and the weak stable foliation $W^{w s}$ of the geodesic flow on the unit tangent bundle $S M$ of $M$. In the course of the proof we point out:

Proposition 2. The weak stable foliation of the geodesic flow on SM has type $\mathrm{III}_{1}$.

Finally we consider the Bowen-Margulis measure $m_{1}$ for the geodesic flow $g_{t}$ on $S M$ [Ma1], [Ma2], [B1], [S], [T], [PP]. It is the measure of maximal entropy for $g_{t}$. Recall that $m_{1}$ is absolutely continuous with respect to the stable and unstable foliations $W^{s}$ and $W^{u}$ of $g_{t}[\mathbf{S}, 2.10]$. In fact, locally $m_{1}$ is a product $\mu^{s} \times \mu^{u} \times d t$ where $\mu^{s}$ and $\mu^{u}$ are measures on $W^{s}$ and $W^{u}$ respectively and $d t$ is Lebesgue measure on $\mathbb{R}$ [Ma2], [PP], [T]. These data determine a lift $m_{2}$ of $m_{1}$ to $S \tilde{M}$. Let $\pi_{1}: S \tilde{M} \rightarrow S$ be given by $\pi_{1}(v)=\gamma_{v}(\infty)$. Set $m=\left(\pi_{1}\right)_{*}\left(m_{2}\right)$. Clearly $m$ is quasiinvariant under $\Gamma$. Note that $m$ is rather awkward since typically the measure $m(A)$ of a set $A$ in $S$ is either 0 or $\infty$. However for $m_{2}$-a.e. $v \in S \tilde{M}$ we can define a nice measure $m_{v}$ on $S$ in the same measure class as $m$ by $m_{v}=\left(\pi_{1}\right)_{*} \mu_{v}^{u}$ where $\mu_{v}^{u}$ is the disintegration of $m_{2}$ to $W_{v}^{u}$.

Projecting $\mu_{v}^{u}$ to the horosphere of $v$ we can think of the $m_{v}$ as projections of measures on horospheres:

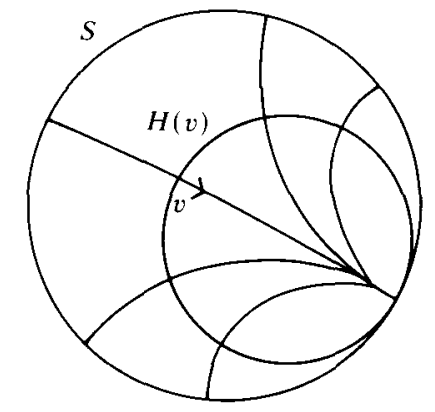

PROPOSITION 3. The $\Gamma$-action on $S$ is ergodic, amenable and has type $\mathrm{III}_{1}$ with respect to the measure class of $\mathrm{m}$.

We expect that the propositions hold true for any compact non-positively curved manifold $M$ of rank 1 [E]. Note that the measure class on the sphere at infinity is still well defined, essentially since the weak stable foliation for the geodesic flow is absolutely continuous.

Let us observe that if $M=\Gamma \backslash G / K$ is a compact locally symmetric space of non-compact type and (real) rank at least 2 , then $\Gamma$ acts amenable but not ergodically on the sphere at infinity $S$ of $G / K$. Indeed, $S$ splits measurably as $S=G / P \times C$ where $P$ is the minimal parabolic subgroup expected in $G$ and $C$ is a Weyl chamber. The canonical measure class on $S$ is the product of Haar measure on $G / P$ and Lebesgue measure on $C$. The action of $\Gamma$ on $S$ is the extension of the $\Gamma$-action on $G / P$ by the identity on $C$. Since an extension of an amenable action is amenable [Z2, theorem 2.4] $\Gamma$ acts amenably on $S$. Clearly the $\Gamma$-action is not ergodic when rank $M \geq 2$.

We would like to thank J. Hawkins for several helpful discussions about type III $_{1}$. 


\section{Proofs}

First we describe the duality between the $\Gamma$-action on $S$ and the weak stable foliation on the unit tangent bundle $S M$ of $M$ : Let $S \tilde{M}$ be the unit tangent bundle of $\tilde{M}$ and let $p_{1}: S \tilde{M} \rightarrow S M$ be the derivative of the covering $\tilde{M} \rightarrow M$. Let $\pi_{1}: S \tilde{M} \rightarrow S$ be given by $\pi_{1}(v)=\gamma_{v}(\infty)$. Set $\mathbb{G}=\Gamma \backslash S$ and let $\pi_{2}: S \rightarrow$ (S) be the projection map. Finally let $p_{2}$ be the projection from $S M$ to the orbit space of the weak stable foliation on $S M$. Since $\pi_{1}$ is $\Gamma$-equivariant this orbit space can be identified with $B$ and the following diagram commutes:

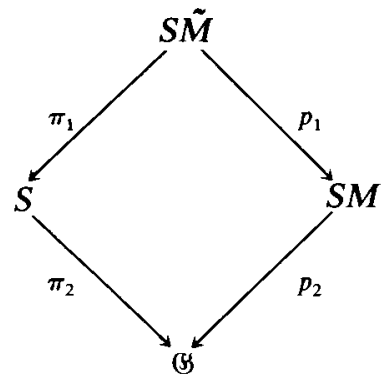

Set $\pi=\pi_{2} \circ \pi_{1}$. Let $\mu$ and $\tilde{\mu}$ be the Liouville measures on $S M$ and $\boldsymbol{S} \tilde{M}$. Clearly, both $p_{1}$ and $\pi_{1}$ are non-singular with respect to $\tilde{\mu}, \mu$ and $\tilde{\mu}, \lambda$.

In [B2, theorem 7, corollary 11] Bowen established:

THEOREM. The weak stable foliation $W^{\text {ws }}$ of a transitive $C^{2}$ Anosov flow $f_{t}$ on a compact manifold is hyperfinite and ergodic. If $f_{t}$ does not have a global cross section then the ratio set of $W^{w s}$ is $[0, \infty)$.

Observe that $\Gamma$ acts ergodically on $S$ because $\pi_{1}$ and $p_{1}$ are non-singular and $W^{\text {ws }}$ is ergodic on $S M$.

Given a map $f: X \rightarrow Y$ let $\sim_{f}$ be the equivalence relation on $X$ defined by $x_{1} \sim_{f} x_{2}$ if $f\left(x_{1}\right)=f\left(x_{2}\right)$.

By Bowen's theorem $\sim_{p_{2}}$ and therefore $\sim_{\pi}$ are amenable equivalence relations. Indeed, amenability and approximate finiteness are equivalent, and extensions of approximately finite relations are approximately finite [M, 5.1, 5.3].

LEMMA 1. Let $X$ and $Y$ be standard measure spaces with measure classes $[\mu]$ and $[\lambda]$, and a non-singular projection map $\pi_{1}: X \rightarrow Y$. Suppose $\pi: X \rightarrow Z$ and $\pi_{2}: Y \rightarrow Z$ are maps such that $\pi=\pi_{2} \circ \pi_{1}$. Then $\sim_{\pi_{2}}$ is amenable if $\sim_{\pi}$ is amenable.

Proof. If $\sim_{\pi_{2}}$ is measurable then so is $\sim_{\pi}$. Let $\alpha$ be a homomorphism of $\sim_{\pi_{2}}$ into the group of isometries Iso $(E)$ of a separable Banach space $E$. Let $y \mapsto K_{y}$ be a measurable field of weak*-compac: sets in $E^{*}$ invariant under $\alpha$ in the sense that $\alpha\left(y_{1}, y_{2}\right)^{*-1} K_{y_{2}}=K_{y_{1}}$ for a.e. $y_{1} \sim_{\pi_{2}} y_{2}$. Composing $\alpha$ and $K$ with $\pi_{1}$ we get a homomorphism of $\sim_{\pi}$ into Iso $(E)$ with invariant field of sets $x \mapsto K_{\pi_{1}(x)}$. Since $\sim_{\pi}$ is amenable there is a fixed point $\phi$ in $K \circ \pi_{1}$, i.e. a Borel funstion $\phi: X \rightarrow E^{*}$ such that $\phi(x) \in K_{\pi_{1}(x)}$ and

$$
\left(\left(\alpha \circ \pi_{1}\right)\left(x_{1}, x_{2}\right)^{*-1}\right) \phi\left(x_{2}\right)=\phi\left(x_{1}\right)
$$

for every $x_{1}, x_{2}$ outside a set of measure 0 (cf. [R] for the technical difference 
between this and a.e. $\left.x_{1} \sim_{\pi} x_{2}\right)$. In particular, we have that $\phi\left(x_{1}\right)=\phi\left(x_{2}\right)$ a.e. if $\pi_{1}\left(x_{1}\right)=\pi_{1}\left(x_{2}\right)$. By this and the non-singularity of $\pi_{1}$ we see that $\phi$ defines a fixed point for $\alpha$ in $K$.

LEMMA 2. The action of $\Gamma$ on $S$ is free a.e.

Proof. Since $M$ is compact all $\gamma \neq 1$ in $\Gamma$ are hyperbolic [BGS, 8.2]. By [BGS, 6.8] each $\gamma \neq 1$ has exactly two fixed points in $S$. Since $\Gamma$ is countable there are only countably many points in $S$ with non-trivial stabiliser.

Recall [ Z1, proposition 3.2] that a free ergodic action of a countable discrete group is amenable if and only if the orbits define an amenable equivalence relation. Hence lemmas 1 and 2 show that $\Gamma$ acts amenably on $S$ with respect to [ $\lambda]$.

Next we prove proposition 2. By [M, 3.3] we need to show that the ratio set is $[0, \infty)$. By Bowen's theorem it suffices to prove that the geodesic flow $g_{t}$ does not admit a global cross section. If it does then $g_{t}$ is a continuous time change of a suspension of a homeomorphism. This is impossible by Arnold's theorem [A, § 23].

Finally we show that the $\Gamma$-action is type $\operatorname{III}_{1}$ or equivalently $[\mathbf{M}, 3.3]$ that the ratio set is $[0, \infty)$. Again we use duality and thus reduce the claim to proposition 2. First observe that $\sim_{\pi}$ is of type $\mathrm{III}_{1}$ by proposition 2 . Since the weak stable foliation $W^{\text {ws }}$ of the geodesic flow on $S \tilde{M}$ is absolutely continuous with respect to the Liouville measure and each unstable leaf $W^{u}(x)$ is transverse to $W^{w s}$ we can disintegrate $\tilde{\mu}=\int_{W^{u}(x)} \mu_{y}^{w s} d \mu_{x}^{u}(y)$, where $x \in S \tilde{M}$ and $\mu_{y}^{w s}$ and $\mu_{x}^{u}$ are measures on $W^{w s}(y)$ and $W^{u}(x)$ respectively. Clearly, $S \tilde{M}=W^{w s}(x) \times W^{u}(x)$ measurably and $\mu$ is equivalent to the product measure $\mu_{x}^{w s} \times \mu_{x}^{u}$. Also note that $\pi_{1}$ becomes the projection to the second factor. Let $\nu_{1}$ be a Haar measure for $\sim_{\pi_{2}}[\mathbf{M}, 3.1]$. The product measure $\nu_{2} \stackrel{\text { def }}{=} \mu_{x}^{u} \times \mu_{x}^{u}$ is a Haar measure for $\sim_{\pi_{1}}$. Then $\nu_{1} \times \nu_{2}$ is a Haar measure for $\sim_{\pi}$. Let $\Delta, \Delta_{1}, \Delta_{2}$ be the modular functions for $\sim_{\pi}, \sim_{\pi_{1}}, \sim_{\pi_{2}}$ respectively [M, 3.1]. Then $\Delta=\Delta_{1} \cdot \Delta_{2}=\Delta_{1}$ since $\Delta_{2} \equiv 1$ by our choice of $\nu_{2}$. Hence the ratio sets $[\mathrm{M}, 3.3]$ of $\Delta_{1}$ and $\Delta$ are the same. Therefore the ratio set of the $\Gamma$-action on $S$ is $[0, \infty)$. This finishes the proof of proposition 1 .

Finally we consider the measure of maximal entropy. By $[\mathbf{S}, 2.10(\mathrm{ii})], W^{w s}$ is hyperfinite with respect to $m_{1}$. Proposition 3 follows from this and [S, 2.13] by the same argument as proposition 1.

\section{REFERENCES}

[A] D. V. Anosov. Geodesic Flows on Closed Riemannian Manifolds of Negative Curvature. Trudy Steklov, 90 (1967), Proceedings of the Steklov Institute of Mathematics, (Petrovskii and Mikolskii, ed.), Amer. Math. Soc. 1969.

[BGS] W. Ballmann, M. Gromov \& V. Schroeder. Manifolds of Non-positive Curvature. Progress in Mathematics vol. 61, Birkhauser 1985.

[B1] R. Bowen. Periodic orbits for hyperbolic flows. Amer. J. Math. 94 (1972), 1-30.

[B2] R. Bowen. Anosov foliations are hyperfinite. Annals of Math. 106 (1977), 549-565.

[E] P. Eberlein. Rigidity problems of manifolds of non-positive curvature. To appear in Proceedings of Conference on Differential Geometry and Global Analysis, Berlin, June 1984.

[Ma1] G. A. Margulis. Applications of ergodic theory to the investigation of manifolds of negative curvature. Funct. Anal. \& Appl. 3 (no. 4), (1969), 335-336. 
[Ma2] G. A. Margulis. Certain measures associated with U-flows on compact manifolds. Funct. Anal. Appl. 4 (no. 1), (1970), 55-67.

[M] C. C. Moore. Ergodic theory and von Neumann algebras. Operator Algebras and Applications, Proceedings of Symposia in Pure Mathematics, vol. 38, part 2, 179-226, Amer. Math. Soc. 1982.

[PP] W. Parry \& M. Pollicott. An analogue of the prime number theorem for closed orbits of Axiom A flows. Annals of Math. 118 (1983), 573-591.

[R] A. Ramsay. Virtual groups and group actions. Adv. in Math. 6 (1971), 253-322.

[S] C. Series. The Poincaré flow of a foliation. Amer. J. Math. 102 (1980), 93-128.

[Su] D. Sullivan. Discrete conformal groups and measurable dynamics. Bull. Amer. Math. Soc. 6 (1982), 57-73

[T] C. H. Toll. A multiplicative asymptotic for the prime geodesic theorem. Thesis, University of Maryland (1984).

[Z1] R. J. Zimmer. Hyperfinite factors and amenable ergodic actions. Invent. Math. 41 (1977), 23-31.

[Z2] R. J. Zimmer. Amenable ergodic group actions and an application to Poisson boundaries of random walks. J. Funct. Anal. 27 (1978), 350-372.

[Z3] R. J. Zimmer. Ergodic theory, group representations and rigidity. Bull. Amer. Math. Soc. 6 (1982), 383-416. 